\title{
Le rôle du bibliothécaire dans la recherche au sein du Département des sciences économiques et administratives de l'Université du Québec à Chicoutimi
}

\author{
Sébastien Leblanc-Proulx ${ }^{a}$ et Marie-Ève Ruest ${ }^{b}$
}

La recherche est l'un des principaux piliers de l'institution universitaire. Sans elle, un bon nombre de découvertes, d'innovations et d'avancements scientifiques ne pourraient avoir lieu. Dans un contexte de conciliation de plus en plus difficile entre l'enseignement et la recherche, le bibliothécaire s'avère un allié important des professeurs.

Cette situation s'apparente bien au Département des sciences économiques et administratives de l'Université du Québec à Chicoutimi (UQAC). Depuis la fondation de l'institution, un bibliothécaire est attitré au développement de collections sur des sujets reliés à ce département. Guy Laberge exécuta cette fonction de 1970 à 1996, suivi de Roger de la Sablonnière jusqu'en 2008. Par la suite, Marie-Ève Ruest, Karine Richer, Luc Charron et Sébastien Leblanc-Proulx ont pris la relève.

Une des caractéristiques qui distingue ce partenariat apparut en 2002, au moment où Roger de La Sablonnière fit preuve d'innovation avec l'appui du directeur de la bibliothèque de l'époque, Gilles Caron. L’idée était de délocaliser le bibliothécaire. Plutôt que de travailler dans la bibliothèque, monsieur de La Sablonnière intégra graduellement le département, à raison d'une demi-journée par semaine, pour ensuite y travailler à mi-temps (De la Sablonnière, Simard et Morency, 2005). Cette idée avait déjà été expérimentée dans quelques universités américaines, comme Virginia Tech, dans les années 1990 (Ruest, 2011). Mais pourquoi donc?

\footnotetext{
a Bibliothécaire, Université du Québec à Chicoutimi

b Bibliothécaire, Université du Québec à Chicoutimi
}

Revue Organisations \& Territoires • Volume 26・ No 1-2 • 2017
Avec la dématérialisation de l'information, l'accessibilité à de la documentation de qualité peut se faire entièrement en ligne. Ainsi, pour plusieurs, il devient contre-productif de se déplacer à la bibliothèque pour faire sa recherche documentaire. Dans cette optique, le bibliothécaire a tout intérêt à se montrer disponible là où les usagers se trouvent afin de leur servir de guide (Shumaker, 2012). En travaillant au département, le bibliothécaire augmente sa visibilité auprès des enseignants et des étudiants. Il devient de ce fait plus facile de créer des liens et de saisir les occasions de collaboration.

Durant les premières années de délocalisation, certains professeurs n'étaient pas encore entièrement familiers avec l'usage de l'ordinateur. Monsieur de la Sablonnière en profita pour leur offrir son aide et les assister dans des tâches rudimentaires (p. ex., envoyer des courriels, imprimer des documents, etc.). En même temps, il entreprit de faire des revues de littérature sur divers sujets demandés par des individus ou des groupes de recherche. Sa crédibilité augmenta rapidement, au point de devenir une référence importante au département dans les domaines de la technologie et de la recherche. Peu à peu, son rôle de bibliothécaire se transforma avec les nouvelles demandes et besoins. C'est d'ailleurs un phénomène qui est commun lors d'une délocalisation, selon Carlton et Kneale (2011).

Cette nouvelle manière d'aborder le travail du bibliothécaire fut à ce point profitable que six des sept autres bibliothécaires disciplinaires de l'UQAC 
l'adoptèrent par la suite. Grâce au modèle organisationnel de la bibliothèque qui permet aux techniciens en documentation de répondre aux besoins en référence documentaire, le transfert se fit en douceur. Aucun service aux usagers n'a été sacrifié dans les lieux de la bibliothèque.

Après la retraite de monsieur de la Sablonnière, Marie-Ève Ruest expérimenta la délocalisation à temps plein avec succès en 2008. Depuis ce temps, le bibliothécaire disciplinaire n'a qu'un seul bureau situé au Département des sciences économiques et administratives.

Bien qu'une tendance s'installe au Canada en ce qui concerne la délocalisation des bibliothécaires (Bedi et Walde, 2017), le modèle reste à ce jour peu utilisé dans les universités québécoises. La plupart d'entre elles proposent encore l'approche traditionnelle du bibliothécaire travaillant à la bibliothèque. Pourtant, l'approche délocalisée a fait ses preuves. À l'UQAC, le nombre de demandes d'information, les collaborations de recherche et les interventions en classe ont augmenté de manière considérable depuis ce changement (Ruest, 2011).

En plus du soutien à l'enseignement que le bibliothécaire offre grâce aux multiples formations données dans les classes, il s'avère un collaborateur indispensable de la recherche scientifique (Carlson et Kneale, 2011). Expert de la recherche documentaire, il peut facilement assister les usagers dans leurs diverses demandes. Faire une recension bibliographique sur un sujet et une époque précise demande du temps et une expertise. Même chose pour instaurer une veille in- formationnelle, créer une base de données bibliographique pour un groupe de recherche, fournir de l'aide à la publication et à la gestion des droits d'auteur, viser la maximisation du repérage de la recherche, et intervenir pour l'obtention d'ISBN, la conservation et la diffusion des données, etc.

Les compétences du bibliothécaire complètent régulièrement celle du chercheur et de ce fait, un temps précieux est épargné lorsque ces deux acteurs travaillent en collaboration. Tout dépendamment du rôle qu'on lui confie, il peut même devenir assez important pour ne plus le considérer comme un assistant ou un consultant, mais plutôt comme un partenaire pour la recherche (Monroe-Gulick, O'Brien et White, 2013). Nous le constatons, entre autres, par sa participation de plus en plus active au sein de groupes de recherche subventionnés (Bedi et Walde, 2017). Par cette offre de service, une amélioration de la productivité sur le plan de la recherche est à portée de main.

Enfin, chaque année, le bibliothécaire disciplinaire au Département des sciences économiques et administratives de l'UQAC travaille de concert avec des dizaines de chercheurs afin de les aider à atteindre leurs objectifs. Cette collaboration s'est construite progressivement depuis plusieurs générations. Désormais délocalisé, il est intégré dans le cursus scolaire des étudiants et est un collaborateur actif pour une panoplie de chercheurs. C'est dans cette perspective que nous souhaitons voir le partenariat entre la bibliothèque Paul-Émile-Boulet et le Département des sciences économiques et administratives se poursuivre, c'est-àdire comme une relation positive et essentielle pour l'enseignement et la recherche.

\section{RÉFÉRENCES}

Bedi, S. et Walde, C. (2017). Transforming roles: canadian academic librarians embedded in faculty research projects. College \& Research Libraries, 78(3). doi: 10.5860/crl.78.3.16590.

Carlson, J. et Kneale, R. (2011). Embedded librarianship in the research context: navigating new waters. College \& Research Libraries News, 72(3), 167-170. doi: 10.5860/crln.72.3.8530.

De la Sablonnière, R., Simard, J. et Morency, M.-A. (2005). Nouvelles approches documentaires dans la pédagogie des sciences économiques et administratives. Documentation et bibliothèques, 51(3), 193-201. doi: 10.7202/1029498ar.

Monroe-Gulick, A., O’Brien, M. et White, G. (2013). Librarians as partners: moving from research supporters to research partners. Dans D.M. Mueller (dir.), Imagine, innovate, inspire: the proceedings of the ACRL 2013 Conference (p. 392-387). Indianapolis, IN: Association of College and Research Libraries (ACRL).

Ruest, M.-È. (2011). Repenser la relation bibliothécaires-professeurs : l'intégration physique d'un bibliothécaire dans un département universitaire. Documentation et bibliothèques, 57(4), 212-218. doi: 10.7202/1028990ar.

Shumaker, D. (2012). The embedded librarian: innovative strategies for taking knowledge where it's needed. Medford, NJ: Information Today Inc. 